\title{
Immunotherapy of metastatic melanoma using genetically engineered GD2-specific T cells
}

\author{
Eric Yvon ${ }^{1}$, Michele Del Vecchio ${ }^{2}$, Barbara Savoldo ${ }^{1}$, Valentina Hoyos ${ }^{1}$, Aurélie Dutour ${ }^{3}$, \\ Andrea Anichini ${ }^{4}$, Gianpietro Dotti ${ }^{1,5,6}$, and Malcolm K. Brenner ${ }^{1,5}$ \\ ${ }^{1}$ Center for Cell and Gene Therapy, Baylor College of Medicine, Texas Children's Hospital, The \\ Methodist Hospital, Houston TX, USA \\ ${ }^{2}$ Medical Oncology Unit, Fondazione IRCCS Istituto Nazionale per lo Studio e la Cura dei Tumori, \\ Milan, Italy. \\ 3INSERM-U590, Centre Léon-Bérard, Lyon, France \\ ${ }^{4}$ Tumor Immunobiology Unit, Istituto Nazionale Tumori, Milan, Italy \\ ${ }^{5}$ Department of Medicine, Baylor College of Medicine, Houston TX, USA \\ ${ }^{6}$ Department of Immunology, Baylor College of Medicine, Houston TX, USA.
}

\begin{abstract}
Purpose-Genetic engineering of human T lymphocytes to express tumor-directed chimeric antigen receptors (CAR) can produce anti-tumor effector cells that bypass tumor immune escape mechanisms that are due to abnormalities in protein-antigen processing and presentation. Moreover, these transgenic receptors can be directed to tumor associated antigens that are not protein derived, such as the ganglioside GD2, which is expressed on a high proportion of melanoma cells.

Experimental design-We generated chimeric T cells specific for the ganglioside GD2 by joining an extracellular antigen-binding domain derived from the GD2-specific antibody sc14.G2a to cytoplasmic signaling domains derived from the TCR $\zeta$-chain, with the endodomains of the costimulatory molecules CD28 and OX40. We expressed this CAR in human T cells and assessed the targeting of GD2+ melanoma tumors in vitro and in a murine xenograft.
\end{abstract}

Results-Upon co-incubation with GD2-expressing melanoma cells, CAR-GD2 T lymphocytes incorporating the CD28 and OX40 endodomains secreted significant levels of cytokines in a pattern comparable to the cytokine response obtained by engagement of the native CD3 receptor. These CAR-T cells had anti-melanoma activity in vitro and in our xenograft model, increasing the survival tumor-bearing animals.

Conclusion-Redirecting human T lymphocytes to a tumor-associated ganglioside GD2 generates effector cells with anti-melanoma activity that should be testable in subjects with disease.

Corresponding author and reprints: Eric YVON, Center for Cell and Gene Therapy, Baylor College of Medicine, 6621 Fannin Street MC

3-3320, Houston, TX 77030, Phone: 832-824-4549, Fax: 832-825-4732, exyvon@txccc.org.

E.Y., M.D., B.S., V.H. and A.D. designed and performed the experiments.

E.Y., B.S., G.D. and M.K.B designed the research and analyzed the data.

E.Y., G.D. and M.K.B. wrote the manuscript.

A.A. provided critical revision of the manuscript.

All authors approved the final version of the manuscript.

The authors declare no competing financial interests. 


\section{Keywords}

Metastatic melanoma; Immunotherapy; Disialoganglioside GD2; chimeric antigen receptor

\section{Translational relevance statement}

T lymphocytes expressing chimeric antigen receptors directed against GD2 can recognize and kill primary melanoma cells, in vitro and in vivo. Administration of such cells to patients with metastatic melanoma may therefore produce benefits.

\section{Introduction}

The rising incidence of cutaneous melanoma and the failure to significantly improve outcomes in metastatic disease has led to increasing interest in immunotherapeutic approaches, since these can be remarkably effective (1-3). Several investigators have focused on targeting tumor associated antigens that fall into the cancer testis antigen (CTA) group, including MAGE, BAGE, GAGE and NY-ESO-1 or the melanocyte differentiation protein (MDP) group, including gp100, Melan-A/MART-1 and tyrosinase, which are widely present on melanoma cells. These studies have used cytotoxic T cell lines $(4,5)$, clones with native (6) or transgenic $\alpha \beta \mathrm{T}$ cell receptors (7) specific for CTA derived peptides which are recognized in association with HLA Class I antigens on the tumor cell surface. It is clear, however, that the heterogeneity of protein antigen expression and presentation in melanoma is a characteristic that helps limit the proportion of patients who are able to respond to such targeted strategies (8). One means of increasing the effectiveness of targeted T cell therapy of melanoma, therefore, may be to use artificial chimeric receptors derived (for example) from the antigen binding domain of a monoclonal antibody (9). When coupled to appropriate intracellular signaling domains, $T$ cells expressing these chimeric antigen receptors (CAR) can kill tumor cell targets (10). They have the advantage of acting in an MHC unrestricted manner, allowing them to target tumor cells in which antigen processing or presentation pathways are disrupted. Moreover, they can be directed to non-peptide antigens on the cell surface, broadening the range of target structures that can be recognized on malignant cells. Hence, CAR-expressing T cells could complement MHC restricted cytotoxic T cells, and increase the overall effectiveness of this cellular immunotherapy.

Many melanoma cells express a range of gangliosides including GD2, GM2, GM3 and GD3 that may be a good choice of target for CAR-T cells, since their expression is highly tissue restricted $(11,12)$. Although these carbohydrate antigens are expressed by both normal melanocytes and melanoma cells, expression is significantly upregulated after malignant transformation of melanocytes $(13,14)$, and associated with changes in the proliferation, migration and metastatic potential of the tumor cells (15). Moreover, natural or vaccineinduced antibodies to gangliosides in melanoma patients have been correlated with improved disease relapse-free survival $(16,17)$.

We now show that the overexpression of GD2 by human primary melanoma cells allows these cells to be targeted in vitro and in vivo by GD2 CAR-expressing primary T cells, and that incorporation of endodomains from both CD28 and OX40 molecules (18) mediates costimulation of the $\mathrm{T}$ lymphocytes, inducing $\mathrm{T}$ cell activation, proliferation and cytotoxicity against GD2+ melanoma cells. 


\section{Materials and Methods}

\section{Establishment of cell lines}

After informed consent, tumor biopsies (from metastatic skin lesions) were obtained from 5 patients with stage III or later melanoma. The tumor tissue was minced and the fragments resuspended in $30 \mathrm{ml}$ of digestion medium containing DNAse at 30U/ml, hyaluronidase at $0.1 \mathrm{mg} / \mathrm{ml}$ and collagenase at $1 \mathrm{mg} / \mathrm{ml}$ (all from Sigma-Aldrich, St Louis, MO), in complete medium prepared as follow: DMEM (Cambrex, Pittsburg, PA) supplemented with $10 \%$ of heat inactivated fetal calf serum (FCS; HyClone, Logan, UT), 200UI/ml penicillin, 200mg/ml streptomycin, $100 \mathrm{mg} / \mathrm{ml}$ gentamycin (Invitrogen, Carlsbad, CA) and 2mM GlutaMAX ${ }^{\mathrm{TM}}$ (Invitrogen). After $4 \mathrm{hrs}$ incubation at $37^{\circ} \mathrm{C}$ in $5 \% \mathrm{CO}_{2}$, the cell suspension supernatant (free of tissue debris) was collected, transferred to a new tube and then centrifuged at $400 \mathrm{xg}$ for $5 \mathrm{~min}$. Cells were re-suspended in a 6 well plate in fresh complete medium containing $1 \mathrm{mM}$ sodium pyruvate (Invitrogen), and cultured at $37^{\circ} \mathrm{C}$ in $5 \% \mathrm{CO}_{2}$. Culture medium was renewed every $72 \mathrm{~h}$. At day 6 , the antibiotics present in the complete medium were reduced to 100UI/ $\mathrm{ml}$ penicillin and $100 \mathrm{mg} / \mathrm{ml}$ streptomycin. When tumor cells reached confluence, they were transferred to a T25 flask for further amplification. The established tumor cell lines (CLB, SENMA, Plaode, RR-371953 and P1143) were characterized by FACS analysis (MCSP and GD2) and immunofluorescence (gp100, MAGE-1 and MART-1). We used low passage number $(<20)$ of the primary melanoma cell lines our in vitro and in vivo experiments.

Normal mesenchymal stem cell (MSCs) and normal skin fibroblast were generated in our laboratory as previously described $(19,20)$ and the K562 cell line was obtained from American Type Culture Collection (ATCC, Rockville, MD). All cell lines were maintained in RPMI (Hyclone) supplemented with $10 \%$ heat inactivated FCS, 100UI $/ \mathrm{ml}$ penicillin, $100 \mathrm{mg} / \mathrm{ml}$ streptomycin, $1 \mathrm{mM}$ Sodium Pyruvate (Invitrogen) and 2mM GlutaMAX ${ }^{\mathrm{TM}}$. Six established melanoma cell lines, isolated from surgical specimens at Istituto Nazionale Tumori, Milan were also used to screen $\mathrm{GD}_{2}$ expression.

\section{Mononuclear cells}

Peripheral blood (PB), obtained after informed consent from normal donors, was processed over Ficoll gradients, and the resulting PB mononuclear cells (PBMCs) were cultured in complete T-cell medium containing 45\% RPMI and 45\% Click's medium (Irvine, CA) supplemented with $10 \%$ heat inactivated FCS, $100 \mathrm{UI} / \mathrm{ml}$ penicillin, $100 \mathrm{mg} / \mathrm{ml}$ streptomycin and $2 \mathrm{mM}$ GLUTAMAX ${ }^{\mathrm{TM}}$.

\section{Retroviral constructs}

The 14g2a scFv sequence was cloned in the SFG retroviral backbone in frame with the human IgG1-CH2CH3 domain, followed by the CD28 and OX40 endodomains and the $\zeta$-chain of the TCR/CD3 complex, to form the $14 \mathrm{~g} 2 \mathrm{a}-\mathrm{CD} 28-\mathrm{OX} 40-\zeta$ (CAR-GD2) construct as previously described (18). We also used vectors encoding the Firefly Luciferase gene (FF-Luc.) or the eGFP protein to track cell survival and proliferation in vivo, as previously described (21). The RD114 retrovirus envelope (RDF plasmid) and the MoMLV gag-pol (PegPam3-e plasmid) were used to engineer the retroviral vectors.

\section{Retrovirus production and transduction}

Transient retroviral supernatants were produced by co-transfection of 293T cells with the PegPam-e, RDF and the desired SFG vectors (CAR-GD2, eGFP or FF-Luc.) using the Fugene6 transfection reagent (Roche, Indianapolis, IN) and used to transduce OKT3 (Ortho Biotech, Bridgewater, NJ) activated PBMCs, as previously described (22). The 4405M, CLB, SENMA and P1143 melanoma cell lines were transfected with retroviral vectors encoding either eGFP 
or FF-Luc. We plated $1 \times 10^{5}$ tumor cells in one well of a 6 well plate and grown to $60-70 \%$ confluency. Culture medium was replaced by the appropriate retroviral supernatant $(1.5 \mathrm{ml}$ per well) and $1 \mu \mathrm{g}$ of polybrene added. When the tumor cells reached confluency, they were trypsinized and plated in a T25 flask. The FF-Luc transduced cells were then selected with puromycin (Sigma-Aldrich, St Louis, MO) at $1 \mu \mathrm{g} / \mathrm{ml}$. The eGFP-transduced tumor cell lines did not require selection as $>98 \%$ of the cells were eGFP+ post retroviral transduction.

\section{Flow cytometry}

Fluorescein isothiocyanate (FITC), Phycoerythrin (PE) or periodin Chlorophyll Protein (perCP) conjugated anti-CD4, -CD8, -CD80 and -CD86 monoclonal antibodies (all from Becton-Dickinson) were used to label lymphocytes, while anti-MCSP-PE (Miltenyi-Biotech Inc., Auburn, CA) and a purified anti-GD2 MAb (Becton-Dickinson Pharmingen) were used to stain the melanoma cells. We added a secondary antibody (RAM-IgG2a+b-E; BectonDickinson) to detect the anti-GD2 (IgG2a) antibody by indirect immunofluorescence. CAR expression by transduced $\mathrm{T}$ lymphocytes was detected using a monoclonal anti-idiotype, 1A7 (TriGem; Titan, South San Francisco, CA) followed by staining with the secondary antibody RAM-IgG1-PE (Becton-Dickinson) (23). The proliferation of non-transduced and transduced $\mathrm{T}$ cells, in presence or absence of tumor cells, was evaluated by FACS analysis after labeling $\mathrm{T}$ cells with CFSE (Invitrogen) according to the manufacturer's instructions.

\section{Cytotoxicity assays}

The cytotoxic activity of the non transduced (NT) and CAR-GD2 T lymphocytes was evaluated in a standard ${ }^{51} \mathrm{Cr}$ release assay, as previously described $(18,22)$. We evaluated isotope release at 6 and 18 hours in cultures with effector-to-target (E:T) ratios of 40:1, 20:1, 10:1 and 5:1, using a $\gamma$-counter (Perkin-Elmer, Shelton, CT).

\section{Co-culture experiments}

Seven days after transduction, NT and CAR-GD2 cells were collected, counted and plated at $5 \times 10^{5}$ cells per well in a 24 well plate at 20:1 ratio with eGFP-expressing (>98\%+) tumor cells. Cytokine release after 24 hours of culture was measured using the CBA array (BD Bioscience) and the percent of CD3+ T cells and eGFP+ tumor cells was evaluated by FACS analysis at day 5 of co-culture, after treatment with $0.5 \%$ trypsin EDTA (Invitrogen) to detach adherent cells.

\section{Xenogeneic SCID mouse model of melanoma}

To assess the in vivo anti-tumor activity of the CAR-GD2 $\mathrm{T}$ lymphocytes, we used a SCID mouse model and the P1143 or $4405 \mathrm{M}$ melanoma line expressing FF-Luciferase. SCID mice (8-9 weeks old) were sublethally irradiated ( $250 \mathrm{rad}$ ) and injected i.v. with $2 \times 10^{6}$ tumor cells. Tumor cell engraftment was monitored using the IVIS 100 imaging system (Caliper Lifesciences, Hopkinton, MA), and on days 4 and $21,1 \times 10^{7}$ non transduced (NT) or CARGD2 T lymphocytes were injected i.v. Animals were imaged weekly to evaluate tumor growth and photon emission from luciferase-expressing cells was quantified using the "Living Image" software provided with the IVIS system (Caliper Life sciences). Briefly, after drawing a region of interest (ROI) over the tumor region, the intensity of the signal measured was expressed as total photons $/ \mathrm{sec} / \mathrm{cm}^{2}\left(\mathrm{p} / \mathrm{s} / \mathrm{cm}^{2} / \mathrm{sr}\right)$.

\section{Statistical analysis}

For cytotoxicity and cytokine production, results were presented as mean \pm SD and paired Student's $t$ test was used to determine statistical significance. For the bioluminescence results, the signal intensity was log transformed and summarized using mean \pm SD at baseline and multiple subsequent time points for each group of mice. Changes in intensity of signal from 
baseline at each time point were calculated and compared using paired $t$-tests or Wilcoxon signed-ranks test. $P$ values $<0.05$ were considered statistically significant.

\section{Results}

\section{Expression of GD2 by primary melanoma cells}

To confirm that GD2 would be a potential target for CAR-directed T cell therapy, we dissociated primary melanoma cells from 5 patients after biopsy of cutaneous metastatic melanoma and used an additional 6 established cell lines in our study. Cells from the 5 patients and 4 of the established lines expressed GD2 on immunofluorescence staining, and between $17 \%$ and $95 \%$ of the cells were positive, with variable intensity of expression (Fig. 1). We also measured expression of GD2 on a normal skin fibroblast cell line and confirmed it did not express the ganglioside. To confirm the absence of non-neoplastic cells in the primary culture, we looked at the expression of MCSP on the cells (Supplemental data: Table 1) and to compare the frequency of expression of GD2 with that of other known melanoma tumor associated antigens, we also measured expression of gp100, MART1 and MAGE-1. The percentage range of GD2 positive cells, was comparable to the range of malignant cells expressing these three other melanoma associated antigens cells (Supplemental data: Table 1). All cell populations studied were negative for expression of the co-stimulatory molecules CD80 or CD86 (data not shown).

\section{T cells expressing a GD2-specific chimeric receptor kill GD2+ melanoma cell lines}

$\mathrm{T}$ cells from 4 healthy donors were transduced with a vector encoding the $14 \mathrm{~g} 2 \mathrm{a}-$ single chain antibody linked to $\zeta$ and to the endodomains of the two co-stimulatory molecules CD28 and OX40, which enhance the activation, proliferation and cytotoxicity of $\mathrm{T}$ cells produced by the CAR after antigen engagement (18). Five days after transduction, we measured the expression of GD2 specific CAR by flow cytometry using the anti-14g2a idiotypic antibody 1A7.and found that $95 \%$ of cells transduced with the $14 \mathrm{~g} 2 \mathrm{a}-\mathrm{CD} 28-\mathrm{OX} 40-\zeta$ retroviral vector were CAR positive (range, 93\%-97\%) (Fig. 2A). The CAR-GD2 construct transduced CD4+ and CD8+ $\mathrm{T}$ cell populations with equal efficiency (Fig. 2B). To mimic the range of GD2 expression seen on primary melanoma cells, we measured the ability of CAR-GD2 T cells to kill three melanoma cell lines with different GD2 expression. $\mathrm{P} 1143$ was a high expressor (95\% positive with MFI $=838$ ); SENMA was intermediate (45\% positive with MFI $=884)$; CLB was low $(19 \%$ positive with MFI $=182)$ and finally, the melanoma cell line $4405 \mathrm{M}$ was used as a GD2 negative tumor cell control. At $6 \mathrm{hrs}$ and $18 \mathrm{hrs},{ }^{51} \mathrm{Cr}$ release assays showed that the anti-tumor activity was proportional to the level of GD2 antigen expression (Fig. 3). As anticipated the CAR-GD2 T cells had little activity against the GD2 negative tumor cell line (4405M), the GD2-NK-cell target line K562 (data not shown) or against normal skin fibroblasts or PBMCs which are also GD2- (Supplemental data: Fig. 1). CAR-GD2 T cells were, however, able to kill an MSC cell line positive for GD2 (95\% positive with MFI = 799) (Supplementary data: Fig. 1). Of note, such cross reactivity with GD2+ normal MSC has not produced discernible adverse effects in any clinical trial of GD2 monoclonal antibodies in patients with neuroectodermal tumors $(24,25)$ or in a phase I study of CAR-GD2 T cells in patients with neuropblastoma.(26).

\section{CAR-GD2 expressing T cells secrete cytokines upon stimulation with GD2-expressing melanoma cells}

Functional activation of CAR-GD2 expressing T cells following their exposure to GD2+ melanoma cells was measured by cytokine release assay. As described above, GD2+ target cells were killed by T cells expressing the CAR and significant IL-2, IL-5, IFN- $\gamma$ and TNF- $\alpha$ release occurred during co-culture with the three melanoma lines. As figure 4 shows, the quantity of IL-2, IL-5, IFN- $\gamma$ and TNF- $\alpha$ secreted by the CAR-GD2 T cells after 24 hours of 
culture, correlated with the level of GD2 expression on the target cells, and was highest for P1143 and lowest for CLB. Neither IL-4 nor IL-10 was detected in the supernatants of stimulated cells.

\section{CAR-GD2 induces sustained killing and clonal expansion in co-culture experiments}

We next determined whether the killing and cytokine release mediated by CAR-GD2 T cells could lead to CAR-T cell proliferation and tumor cell eradication in vitro in a 5 days co-culture experiment. We used CFSE labeled control or CAR-GD2 T cells, to determine whether CAR stimulation by CAR-GD2-expressing $\mathrm{T}$ cells induces effector $\mathrm{T}$ cell proliferation. Non transduced T cells proliferated only in the presence of exogenous IL-2 $(100 \mathrm{U} / \mathrm{ml})$, while proliferation of CAR-expressing T cells increased in response to all three GD2 expressing tumor cell lines, irrespective of whether these tumor cells expressed high, intermediate or low levels of GD2 (Supplemental data: Fig. 2). To discover if these expanded CAR-GD2 T cells were functional, we labeled tumor cells with eGFP and co-cultured them at the CAR T cell: tumor cell ratio of 20:1 in the absence of IL2. After 5 days of culture, we enumerated viable GFP+ cells by flow cytometric analysis. Figure 5 shows that viable tumor cells were eradicated in co-cultures with $\mathrm{T}$ cells expressing CAR-GD2 but not in co-cultures with non transduced T cells. Hence CAR-GD2 T cells proliferate in vitro in response to the GD2 antigen and eradicate melanoma cells that express the antigen. As expected, the GD2 negative cell line 4405M was not killed in the 5 day co-culture experiment, demonstrating GD2 antigen recognition is essential for the activity of CAR-GD2 expressing T cells.

\section{Adoptive transfer of GD2-specific T cells provide anti-tumor effect in a xenogeneic SCID model}

We next measured the anti-tumor activity of CAR-GD2 T cells in vivo. To monitor tumor cells in vivo, we expressed the firefly luciferase (FFLuc) gene in $4405 \mathrm{M}$ and P1143 cells, together with the puromycin-resistance gene. After puromycin selection, we injected $2 \times 10^{6}$ FFLucP1143 or 4405M tumor cells i.v. into SCID mice. After 4 days, FFLuc expression was evaluated by bioluminescence imaging and the mice were divided into 3 groups which received non transduced (NT) T cells or T cells expressing CAR-GD2 at $1 \times 10^{7}$ i.v and finally a group that received tumor cells alone. A second injection of NT or CAR-GD2 T cells was given at day 21 and we measured luciferase signal every week in the 10 mice of each of the groups. Figure 6A shows 5 representative mice from the NT and CAR-GD2 T cells group and demonstrates that tumor grew rapidly in the lungs of mice receiving NT T cells. By contrast, the tumors in mice receiving T cells expressing CAR-GD2 diminished within 48-72 hrs of injection and luciferase-derived remained largely absent in the group receiving CAR-GD2 T cells. While the survival of the mice receiving the tumor cells alone or tumor cells plus NT T cells was 68 \pm 6 days and $72 \pm 12$ days respectively $(\mathrm{p}=0.03), 80 \%$ of the mice from the group receiving CAR-GD2 T cells were still alive at day 100 and shown a significant survival advantage when receiving CAR-GD2 specific T cells $(\mathrm{p}=0.006)$ (Fig. 6B). Finally, we observed no tumor regression when CAR-GD2 T cells were infused in mice bearing GD2- 4405M tumor cells (Fig. 6A).

\section{Discussion}

We have shown that the ganglioside antigen GD2 is expressed on the majority of primary melanoma cell lines, and that $\mathrm{T}$ cells engineered to express a CAR directed to this antigen are able to recognize and lyse GD2+ melanoma target cells in vitro and in a SCID mouse model in vivo. The transgenic receptor construct included the signaling endodomains of the CD28 and OX40 co-stimulatory molecules, and redirected $\mathrm{T}$ cells showed activation, proliferation and cytokine release after $\mathrm{T}$ cell receptor engagement by GD2. 
Melanoma has long been a target for cellular immunotherapies directed to the tumor associated antigens (TAA) expressed by the malignant cells. While earlier clinical research focused on reinfusion of expanded tumor infiltrating lymphocytes, efforts have recently been directed against the tumor testis series of antigens such as MAGE and MART-1, by generation of T cells expressing conventional $\alpha \beta$-T-cell receptors (TCR) specific for these antigens. These receptors recognize peptide fragments in association with MHC class I molecules, and are therefore restricted in their patient range to individuals with the appropriate MHC polymorphism. Moreover they are unable to recognize tumor sub-clones in which the antigen processing machinery is deficient. $\mathrm{T}$ cells that express synthetic or chimeric receptors that recognize unprocessed structures on the cell surface may thus have an advantage over T cells whose tumor reactivity is mediated through their native receptor.

It has been known for sometime that the ganglioside GD2 is expressed by tumors derived from neuroectoderm, including neuroblastoma, sarcoma, and small lung cancer. This tumor associated carbohydrate antigen (TACA) is also expressed by many melanoma cells (27), where it is involved in cell adhesion and may contribute to metastasis (28). Although GD2 is present on the surface of many melanoma cells, it is absent on most normal tissue, with only limited expression in brain and on peripheral nerves, making this ganglioside an attractive target for adoptive cell therapy in metastatic melanoma (29).

GD2 monoclonal antibodies have already been used with benefit in patient with other GD2 positive malignancies, such as neuroblastoma, but melanoma cells have more variable (and usually lower) expression of the antigen. Hence the benefits of GD2 antibody infusion in melanoma have been limited $(24,25)$. The level of GD2 expression on melanoma cells is, however, evidently sufficient to produce a cytotoxic response from $\mathrm{T}$ cells expressing the same $\mathrm{MAb}$ binding site in the form of a chimeric $\mathrm{T}$ cell receptor. We saw complete killing of the tumor cells even when GD2 expression was low, consistent with previous observations that even tumor cells with dim expression of the targeted antigen can be eliminated by CARmodified T cells (30). The killing of cells that are resistant to antibodies of the same specificity may related to the improved avidity of multiple antibody-derived binding domains when they are arrayed on a cell surface rather than existing as bivalent molecules in solution, or it may reflect a superior cytolytic activity of $\mathrm{T}$ effector cells compared to antibody (31). Hence, tumor cells with dim antigen expression can be completely eliminated after co-culture experiments, even when short term assays based on ${ }^{51} \mathrm{Cr}$ release assay may produce lower immediate cytotoxicity than tumor cells with high antigen expression. Like most malignancies, melanoma cells lack expression of the $\mathrm{T}$ cell costimulatory molecules required for complete activation of $\mathrm{T}$ lymphocytes that engage tumor associated antigens through their native or chimeric receptors. Hence to optimize T cell triggering and effector function, we coupled the chimeric receptor to costimulatory endodomains to increase $\mathrm{T}$ cell survival and expansion. Following CAR engagement, endodomains from single co-stimulatory molecules such as CD28, 4-1BB or OX40 into the CAR, may be sufficient to activate the cellular components of the killing machinery and to produce IL-2 release and T cell proliferation $(32,33)$. We have previously shown, however, that the simultaneous expression in cis of two endodomains such as CD28 and OX40 within a GD2-CAR produces superior T-cell proliferation and effector function than expression of a single co-stimulatory endodomain (18). This benefit likely occurs because both CD28 and OX40 signals are both functional, and produce greater activation of NFKB than either endodomain alone, since they act through two independent pathways (18).

If adoptive transfer of CAR modified T cells in melanoma is to be of clinical value, it will be essential to be able to treat metastatic disease. Accordingly, we studied the effects of the CARGD2 T cells in a xenograft lung metastatic model. Human T cells expressing the $14 \mathrm{~g} 2 \mathrm{a}-\mathrm{CD} 28$ OX40- $\zeta$ CAR produced significant anti-tumor activity in this model, but was unable to completely eradicate the disease. This incomplete benefit may reflect the difficulties of 
sustaining human $\mathrm{T}$ cell function and trafficking in a xenogeneic environment, or it may also represent the limitations of even the combination of CD28 and OX40 endodomains, which on their own cannot completely recapitulate the temporo-spatial features of the co-stimulatory events required to sustain T cell activation physiologically $(26,34)$.

In summary we have demonstrated that GD2 on melanoma cells is a potential target for CAR$\mathrm{T}$ cells. We believe that administration of such cells may usefully complement other cellular immunotherapies and biotherapies for this disease.

\section{Supplementary Material}

Refer to Web version on PubMed Central for supplementary material.

\section{Acknowledgements}

This work was supported by NIH grant PO1 CA94237. B.S. is supported by NIH grant 1RO1CA131027 and by a Leukemia and Lymphoma Society Translational Research grant. G.D. is supported by the Doris Duke Charitable Foundation/Clinical Scientist development award and by a Leukemia and Lymphoma Society Translational Research grant. We would like to thank Dr. Parmiani for critical revision of the manuscript.

\section{References}

1. Jemal A, Siegel R, Ward E, et al. Cancer statistics, 2008. CA Cancer J Clin 2008;58:71-96. [PubMed: 18287387]

2. Bajetta E, Del Vecchio M, Bernard-Marty C, et al. Metastatic melanoma: chemotherapy. Sem Oncol 2002;29:427-445.

3. Rosenberg SA, Restifo NP, Yang JC, Morgan RA, Dudley ME. Adoptive cell transfer: a clinical path to effective cancer immunotherapy. Nat Reviews 2008;8:299-308.

4. Mackensen A, Meidenbauer N, Vogl S, Laumer M, Berger J, Andreesen R. Phase I study of adoptive T-cell therapy using antigen-specific CD8+ T cells for the treatment of patients with metastatic melanoma. J Clin Oncol 2006;24:5060-5069. [PubMed: 17075125]

5. Butler MO, Lee JS, Ansen S, et al. Long-lived antitumor CD8+ lymphocytes for adoptive therapy generated using an artificial antigen-presenting cell. Clin Cancer Res 2007;13:1857-1867. [PubMed: 17363542]

6. Rosenberg SA, Dudley ME. Cancer regression in patients with metastatic melanoma after the transfer of autologous antitumor lymphocytes. PNAS 2004;101:14639-14645. [PubMed: 15381769]

7. Morgan RA, Dudley ME, Wunderlich JR, et al. Cancer regression in patients after transfer of genetically engineered lymphocytes. Science 314:126-129.

8. Ohnmacht GA, Marincola FM. Heterogeneity in expression of human leukocyte antigens and melanoma-associated antigens in advanced melanoma. J Cell Physiol 2000;182:332-338. [PubMed: 10653599]

9. Pule M, Finney H, Lawson A. Artificial T-cell receptors. Cytotherapy 2003;5:211-226. [PubMed: 12850789]

10. Haynes NM, Trapani JA, Teng MW, et al. Single-chain antigen recognition receptors that costimulate potent rejection of established experimental tumors. Blood 2002;100:3155-3163. [PubMed: 12384413]

11. Yun CO, Nolan KF, Beecham EJ, Reisfeld RA, Junghans P. Targeting of T lymphocytes to melanoma cells through chimeric anti-GD3 immunoglobulin T-cell receptors. Neoplasia 2000;2:449-459. [PubMed: 11191112]

12. Livingston P. Ganglioside vaccines with emphasis on GM2. Semin. Oncol 1998;25:636-645. [PubMed: 9865678]

13. Tsuchida T, Sazton RE, Morton DL, Irie RF. Gangliosides of human melanoma. Cancer 1989;63:1166-1174. [PubMed: 2917320] 
14. Albino AP, Sozzi G, Nanus DM, Jhanwar SC, Houghton AN. Malignant transformation of human melanocytes: induction of a complete melanoma phenotype and genotype. Oncogene 1992;7:23152321. [PubMed: 1437153]

15. Ravindranath MH, Muthugounder S, Presser N. Ganglioside signatures of primary and nodal metastatic melanoma cell lines from the same patient. Melanoma Res 2008;18:47-55. [PubMed: 18227708]

16. Livingston PO, Wong GY, Adluri S, et al. Improved survival in stage III melanoma patients with GM2 antibodies: a randomized trial of adjuvant vaccination with GM2 ganglioside. J. Clin. Oncol 1994;12:1036-1044. [PubMed: 8164027]

17. Ragupathi G, Livingston PO, Hood C, et al. Consistent antibody response against ganglioside GD2 induced in patients with melanoma by a GD2 lactone-keyhole limpet hemocyanin conjugate vaccine plus immunological adjuvant QS-21. Clin. Cancer Res 2003;9:5214-5220. [PubMed: 14614001]

18. Pule MA, Straathof KC, Dotti G, Heslop HE, Rooney CM, Brenner MK. A chimeric T cell antigen receptor that augments cytokine release and supports clonal expansion of primary human $\mathrm{T}$ cells. Mol Ther 2005;12:933-941. [PubMed: 15979412]

19. Yvon ES, Vigouroux S, Rousseau RF, et al. Overexpression of the Notch ligand, Jagged-1, induces alloantigen-specific human regulatory T cells. Blood 2003;102:3815-3821. [PubMed: 12842995]

20. Gottschalk S, Edwards OL, Sili U, et al. Genearting CTLs against subdominant Epstein-Barr virus LMP1 antigen for the adoptive immunotherapy of EBV-associated malignancies. Blood 2003;101:1905-1912. [PubMed: 12411306]

21. Savoldo B, Rooney CM, Di Stasi A, et al. Epstein Barr virus-specific cytotoxic T lymphocytes expressing the anti-CD30 artificial chimeric T-cell receptor for immunotherapy of Hodgkin disease. Blood 2007;110:2620-2630. [PubMed: 17507664]

22. Vera J, Savoldo B, Vigouroux S, et al. T lymphocytes redirected against the kappa light chain of human immunoglobulin efficiently kill mature B lymphocyte-derived malignant cells. Blood 2006;108:3890-3897. [PubMed: 16926291]

23. Rossig C, Bollard CM, Nuchtern JG, et al. Targeting of G(D2)-positive tumor cells by human T lymphocytes engineered to express chimeric T-cell receptor genes. Int J Cancer 2001;94:228-236. [PubMed: 11668503]

24. Saleh MN, Khazaeli MB, Wheeler RH, et al. Phase I trial of the murine monoclonal anti-GD2 antibody 14G2a in metastatic melanoma. Cancer Research 1992;52:4342-4347. [PubMed: 1643631]

25. Murray JL, Cunningham JE, Brewer H, et al. Phase I trial of murine monoclonal antibody 14G2a administred by prolonged intravenous infusion in patients with neuroectodermal tumors. J. Clin. Oncol 1994;12:184-193. [PubMed: 8270976]

26. Pule MA, Savoldo B, Myers GD, et al. Virus-specific T cells engineered to coexpress tumor-specific receptors: persistence and antitumor activity in neuroblastoma patients. Nat. Med 2008;14:12641270. [PubMed: 18978797]

27. Cheresh DA, Harper JR, Schulz G, Reisfeld RA. Localization of the gangliosides GD2 and GD3 in adhesion plaques and on the surface of the human melanoma cells. Proc. Natl. Acad. Sci. USA 1984;81:5767-5771. [PubMed: 6385004]

28. Hakomori S. Tumor-associated carbohydrate antigens defining tumor malignancy: basis for development of anti-cancer vaccines. Adv. Exp. Med. Biol 2001;491:369-402. [PubMed: 14533809]

29. Hersey P, Jamal O, Henderson C, Zardawi I, D'Alessandro G. Expression of the gangliosides GM3, GD3 and GD2 in tissue sections of normal skin, naevi, primary and metastatic melanoma. Int. J. Cancer 1988;41:336-343. [PubMed: 3346097]

30. Vera J, Savoldo B, Vigouroux S, et al. T lymphocytes redirected against the kappa light chain of human immunoglobulin efficiently kill mature B lymphocyte-derived malignant cells. Blood 2006;108:3890-3897. [PubMed: 16926291]

31. Weijtens ME, Hart EH, Bolhuis RL. Functional balance between T cell chimeric receptor density and tumor associated antigen density: CTL mediated cytolysis and lymphokine production. Gene Ther 2000;7:35-42. [PubMed: 10680014]

32. Willemsen RA, Ronteltap C, Chames P, Debets R, Bolhuis RLH. T Cell Retargeting with MHC Class I-Restricted Antibodies: The CD28 Costimulatory Domain Enhances Antigen-Specific Cytotoxicity and Cytokine Production. J. Immunol 2005;174:7853-7858. [PubMed: 15944290] 
33. Imai C, Mihara K, Andreansky M, et al. Chimeric receptors with 4-1BB signaling capacity provoke potent cytotoxicity against acute lymphoblastic leukemia. Leukemia 2004;18:676-684. [PubMed: 14961035]

34. Heemskerk MHM, Hoogeboom M, Hagedoorn R, Kester MGD, Willemze R, Falkenburg FJH. Reprogramming of virus-specific $\mathrm{T}$ cells into Leukemia-reactive $\mathrm{T}$ cells using $\mathrm{T}$ cell receptor gene transfert. J. Exp. Med 2004;199:885-894. [PubMed: 15051765] 


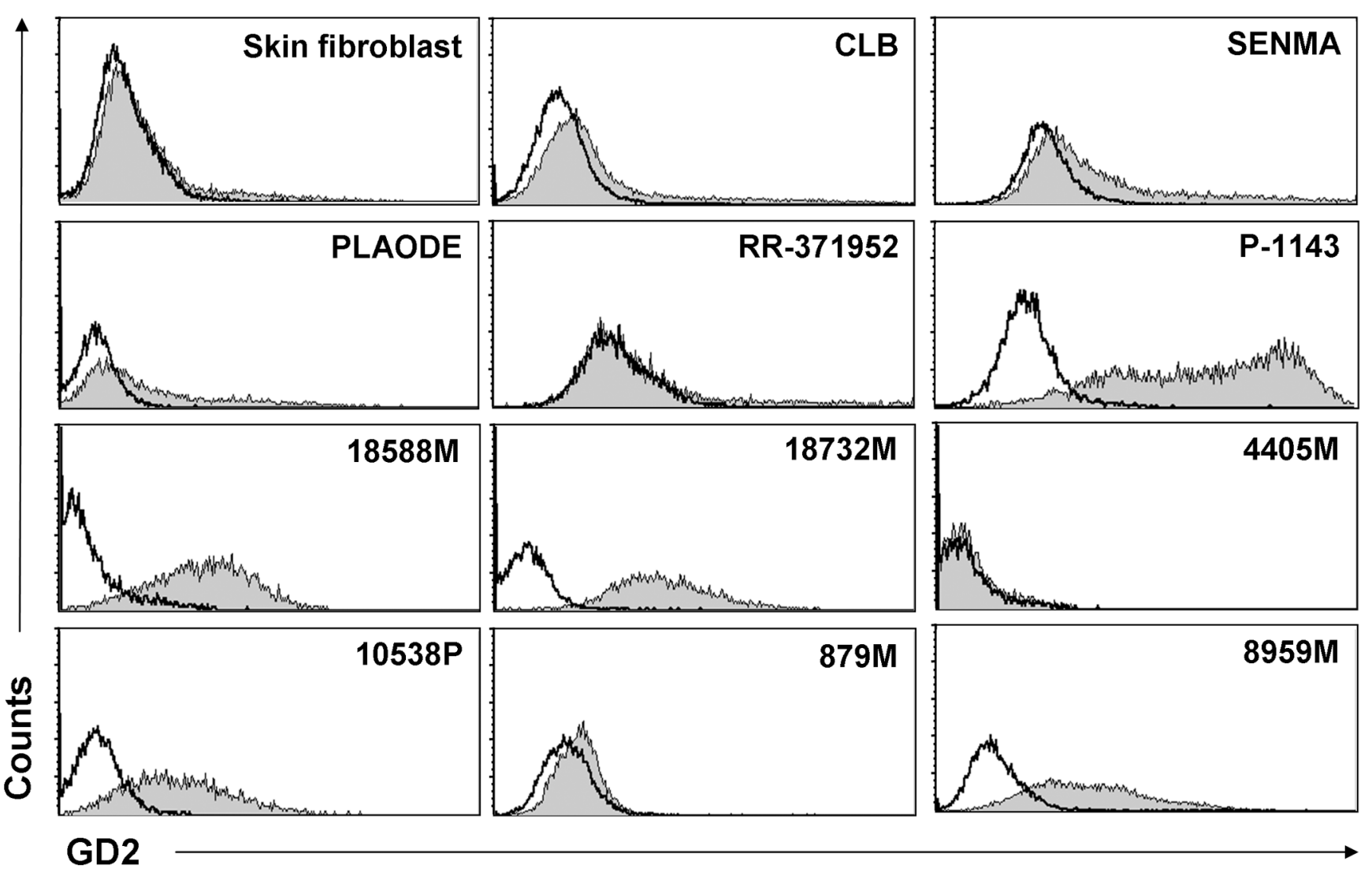

Fig. 1. Expression of GD2 antigen in human melanoma cell lines

The expression of GD2 was evaluated by FACS analysis in 11 melanoma cell lines. Six (45\%) and $3(27 \%)$ tumor cell lines showed GD2 expression at intermediate/high $(++/+++)$ or low levels (+), respectively. In 2 tumor cell lines (18\%) GD2 was undetectable. A GD2- normal skin fibroblast line was used as a negative control for GD2 expression. Open histograms represent the isotype control of the GD2 staining (grey histograms). 
NT
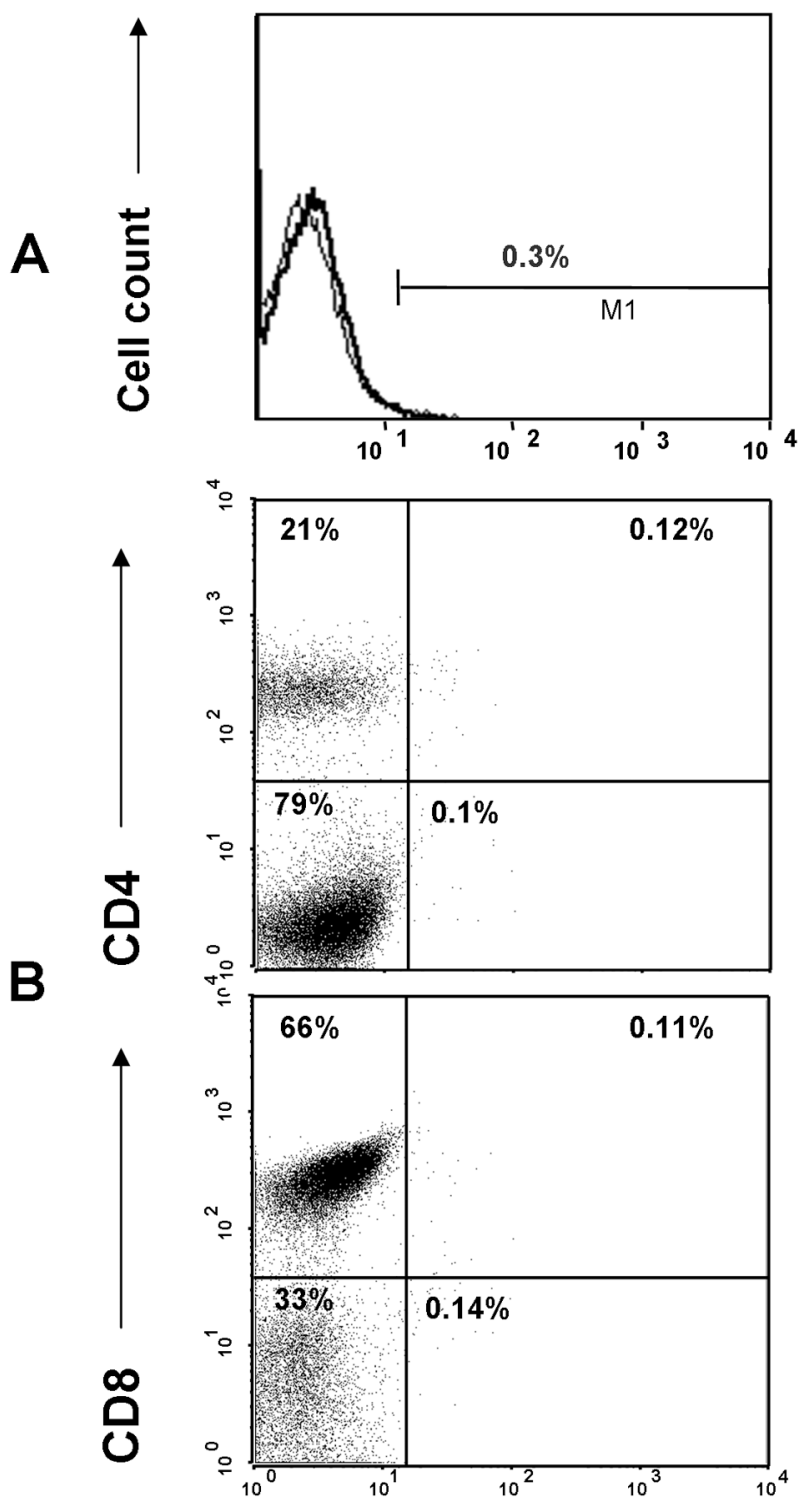

CAR-GD2
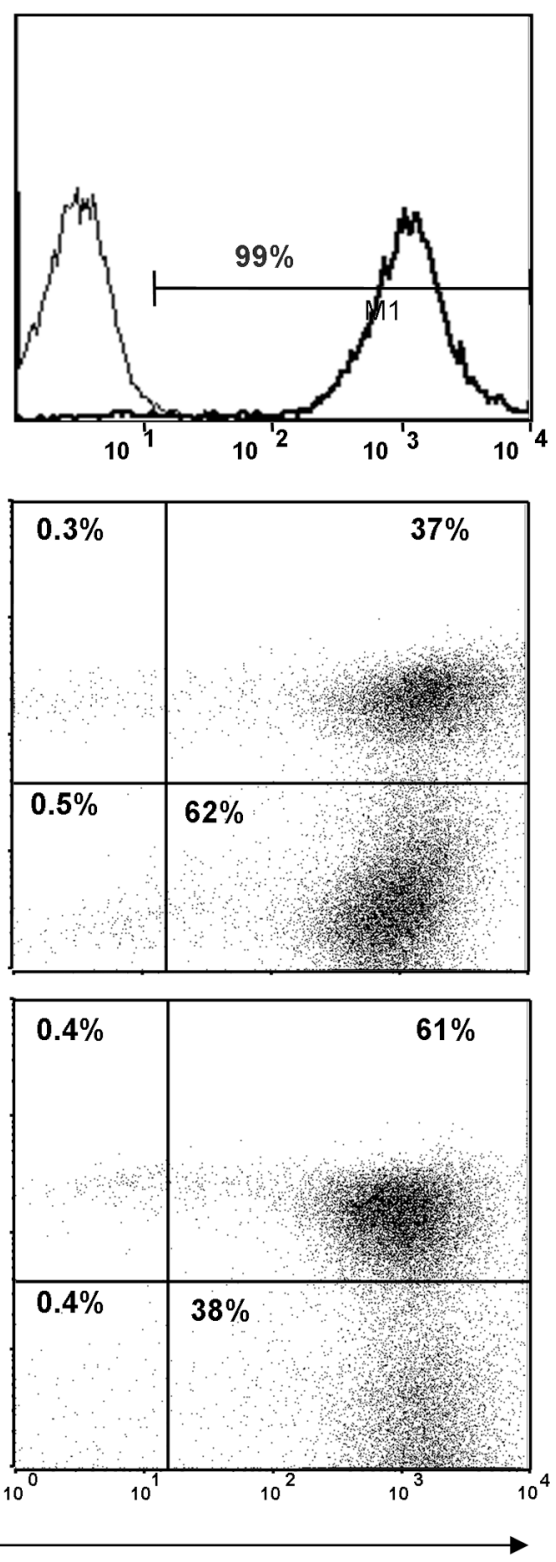

Fig. 2. T-lymphocytes can be genetically modified to express CARs targeting GD2

Activated T lymphocytes were genetically modified to express CAR-GD2. Panel A illustrates the expression of CAR-GD2 as assessed by FACS analysis using a specific 14.g2a anti-idiotype antibody (1A7). The graph shows a representative expression of CAR-GD2 from 4 different transduced T cell lines. Panel B shows that both CD4+ and CD8+ T lymphocytes expressed the CAR-GD2 after gene transfer. 


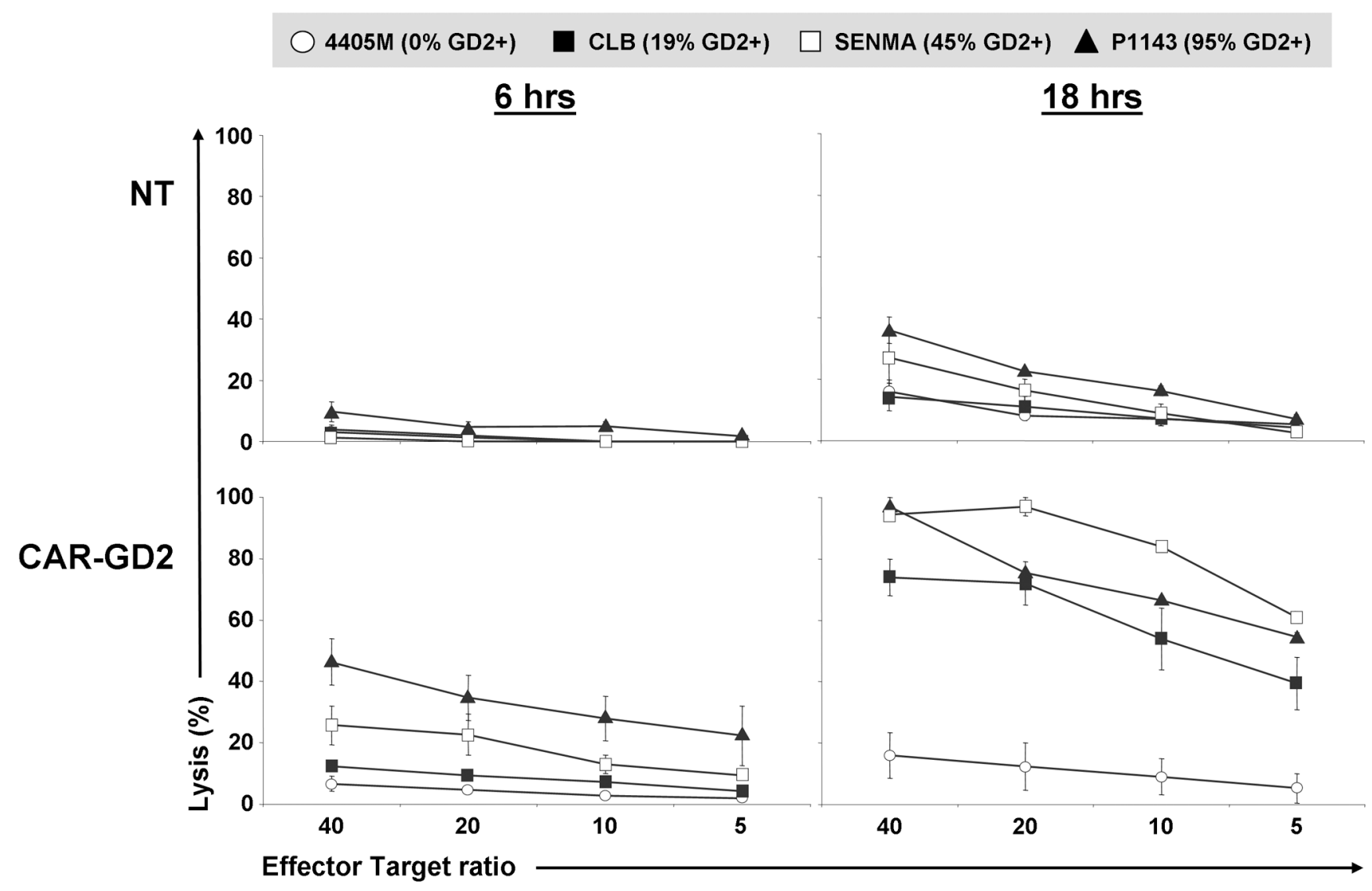

Fig. 3. T-lymphocytes redirected to express CAR-GD2 kill GD2+ melanoma cell lines We used a ${ }^{51} \mathrm{Cr}$ release assay to evaluate the cytotoxic activity of T lymphocytes expressing CAR-GD2 and non transduced (NT) T cells. Target cells were melanoma lines with absent GD2 (4405M) or low (CBL), intermediate (SEMMA) or high (P1143) GD2 expression. Panels $\mathrm{A}$ and $\mathrm{B}$ illustrate the mean and SD of ${ }^{51} \mathrm{Cr}$ release from $4 \mathrm{~T}$ cell lines after 6 and 18 hours incubation, respectively. 


\section{M (0\% GD2+) $\square$ CLB (19\% GD2+)}

SENMA (45\% GD2+)

P1143 (95\% GD2+)
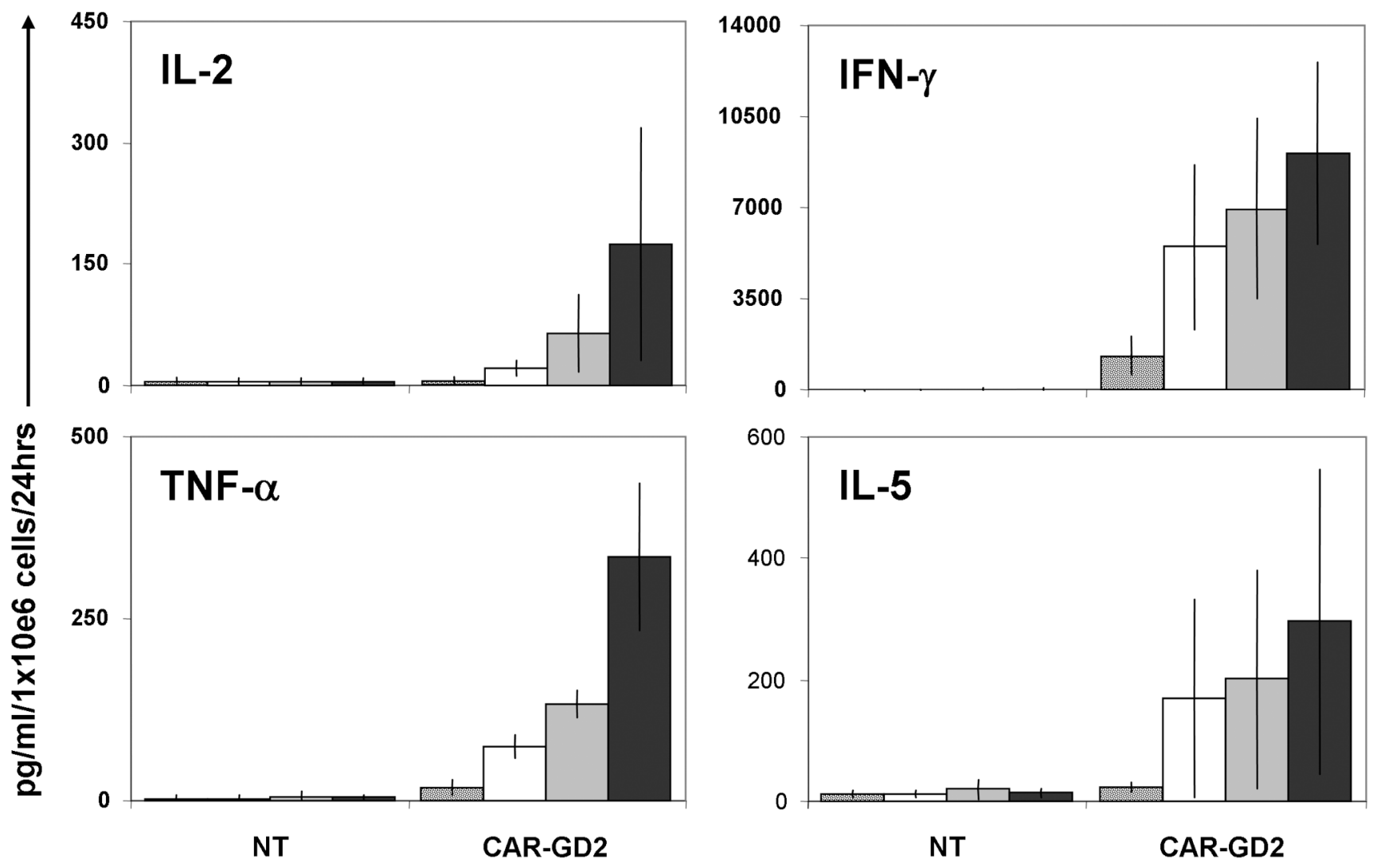

Fig. 4. T-lymphocytes expressing GD2-CAR produce Th1 and Th2 cytokines in response to GD2+ melanoma cell lines

T-lymphocytes transduced with CAR-GD2 or non transduced (NT) T cells were co-cultured (ratio T-lymphocytes:tumor cells of 20:1) with four different melanoma cell lines either negative for GD2 (4405M) or expressing dim (CBL), intermediate (SEMMA) or high (P1143) levels of GD2. Culture supernatant was collected 24 hours later and the production of IL-2, IL5, IFN $\gamma$ and TNF $\alpha$ measured using a CBA assay. Neither IL4 nor IL10 were detected in the 24 hour supernatants. The results of four experiments are presented. 
A

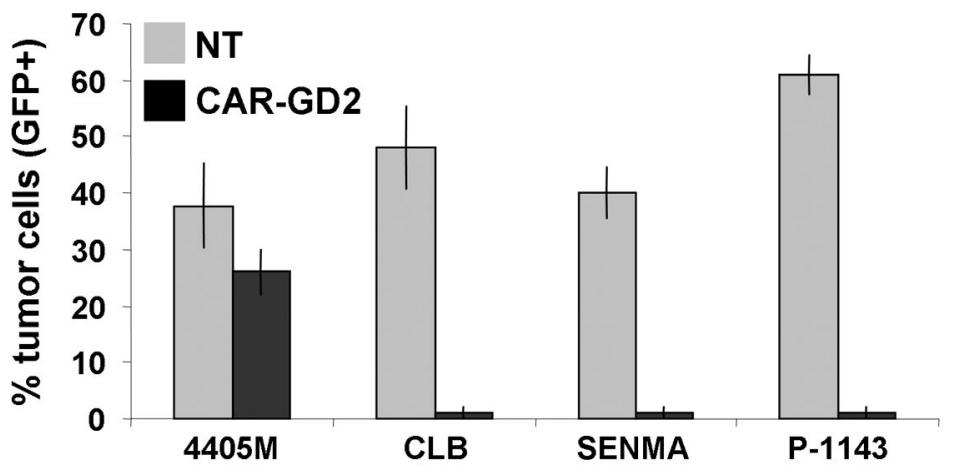

$4405 M=0 \%$ GD2+

CLB $=19 \%$ GD2+

SENMA $=45 \%$ GD2+

$P 1143=95 \%$ GD2+

B
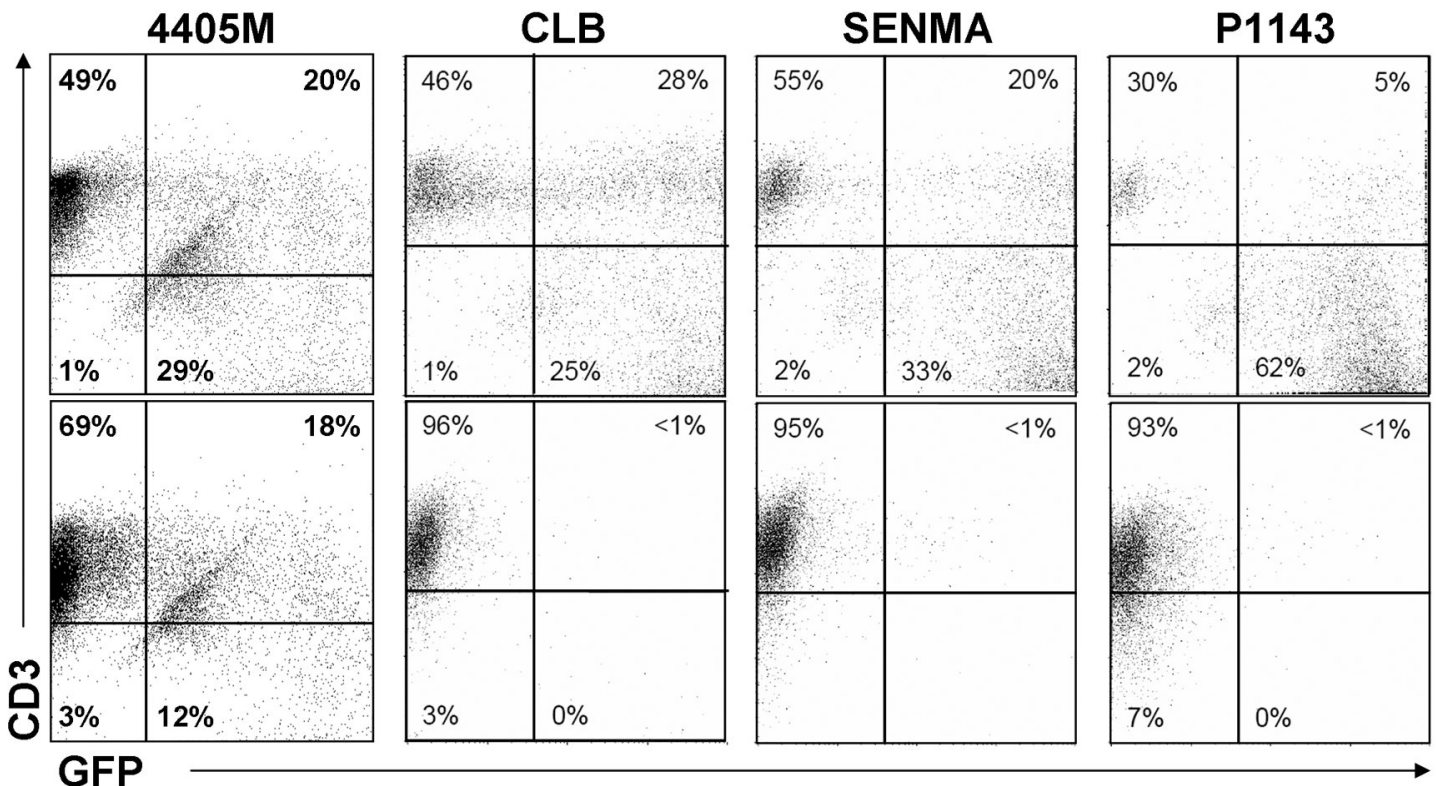

Fig. 5. T-lymphocytes redirected to express CAR-GD2 eliminate GD2+ melanoma cell lines in vitro To evaluate the capacity of T lymphocytes expressing CAR-GD2 to eliminate melanoma cells, we cultured non transduced (NT) or CAR-GD2 transduced T lymphocytes with melanoma cell lines that were GD2- (4405M) or expressed $\operatorname{dim}(\mathrm{CBL})$, intermediate (SEMMA) or high (P1143) levels of the target antigen.. T lymphocytes and melanoma cell lines were plated at 20 to 1 ratio and cultured for 5 days without adding IL2 to the culture. Residual melanoma cells were enumerated by FACS analysis. Panel A illustrates mean and SD of surviving cells expressing GFP for 4 T-cell lines. Panel B shows a phenotypic analysis of co-culture experiments. 
Tumor: P1143 (95\% GD2+)

A
高

NT

7

14

21
CAR-GD2

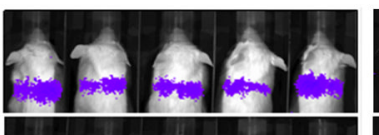

23

28
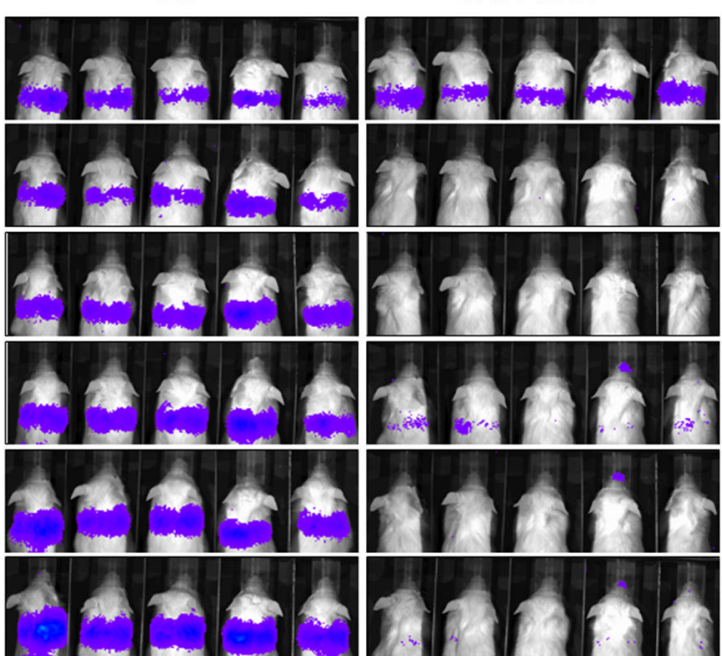

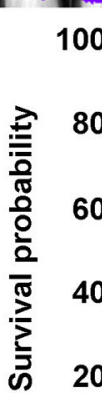

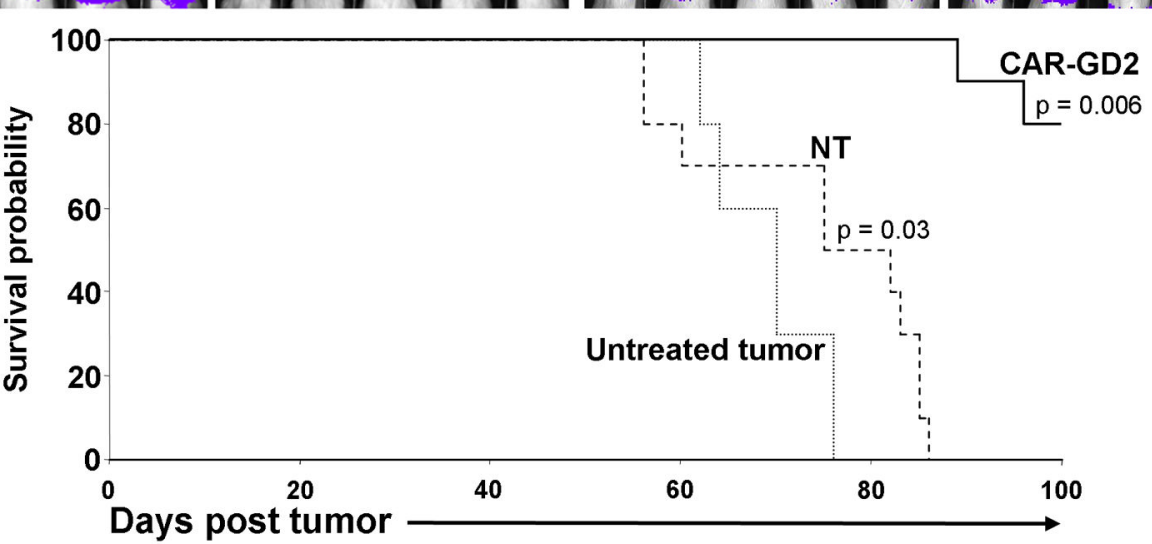

Fig. 6. CAR-GD2 T-lymphocytes control tumor growth in vivo

SCID mice were infused i.v. with $2 \times 10^{6}$ melanoma cells from the cell lines $4405 \mathrm{M}$ (0\% GD2 + ) or P1143 (95\% GD2+) labeled with FFLuc gene. Tumor growth and engraftment was monitored using an in vivo imaging system (Xenogen-IVIS Imaging System). Four and 21 days after tumor infusion, mice were treated with T-lymphocytes CAR-GD2 or non transduced (NT) $\mathrm{T}$ cells $\left(1 \times 10^{7}\right.$ cells/mouse). No exogenous cytokines were injected into the mice. Panel A illustrates the tumor growth measured as light emission in a representative cohort of 5 mice from each group of NT and CAR-GD2 T cells treated animals. Panel B shows the survival curve of mice engrafted with the P1143 (95\% GD2+) tumor cells receiving either tumor alone, NT T cells or CAR-GD2 T lymphocytes.

Clin Cancer Res. Author manuscript; available in PMC 2010 September 15. 